\title{
Prostate cancer incidence in males with Lynch syndrome
}

\author{
Sigurdis Haraldsdottir, MD, MS1, Heather Hampel, MS², Lai Wei, PhD³, Christina Wu, MD¹, \\ Wendy Frankel, MD ${ }^{4}$, Tanios Bekaii-Saab, MD1, Albert de la Chapelle, MD, PhD ${ }^{5}$ \\ and Richard M. Goldberg, MD ${ }^{1}$
}

\begin{abstract}
Purpose: An increased risk of prostate cancer is currently not considered a part of the Lynch syndrome spectrum. The purpose of this study was to retrospectively examine prostate cancer incidence in the Lynch syndrome cohort at the Ohio State University in comparison with that in the general population.

Methods: We included all males diagnosed with Lynch syndrome from June 1998 to June 2012 at the Ohio State University and obtained baseline information including cancer history. If patients had not been seen in the 12 months before June 2012, they were contacted to document changes in their cancer history. We compared prostate cancer incidence among the Lynch syndrome families with that of the general population by using the Surveillance, Epidemiology, and End Results registry 19992009.
\end{abstract}

\section{INTRODUCTION}

Lynch syndrome (LS) is caused by a germline mutation in one of the mismatch repair (MMR) genes, MLH1, MSH2, MSH6, or PMS2. This, followed by a mutation (e.g., chromosomal deletion, point mutation, or epigenetic inactivation by hypermethylation) in a second allele leads to lack of MMR function, causing accumulation of mutations. ${ }^{1}$ This can lead to malignant transformation of cells and tumor formation with a hypermutated phenotype and is manifested by microsatellite instability (MSI) and lack of one or more of the four MMR proteins on staining by immunohistochemistry (IHC). A definitive diagnosis is obtained by germline gene sequencing.

LS increases the risk of several cancers, including colorectal, endometrial, ovarian, gastric, small intestinal, pancreatic, ureteral, brain, and sebaceous gland adenocarcinomas, ${ }^{2}$ and screening for colorectal cancer (CRC) ${ }^{3}$ and prophylactic total abdominal hysterectomy with bilateral salpingo-oophorectomy have been shown to increase survival of these patients. ${ }^{4}$

Prostate and breast cancer are currently not considered part of the LS tumor spectrum. Some studies have revealed an increased incidence of prostate cancer, especially in patients carrying an $\mathrm{MSH} 2$ mutation, ${ }^{5-8}$ whereas others have not shown an increased incidence. ${ }^{2,9,10}$ Two studies have shown a lack of MMR protein expression in prostate cancer tumors in patients
Results: Of the 188 males identified with Lynch syndrome, 11 males were diagnosed with prostate cancer during the study period. The ratio of observed to expected numbers of prostate cancer cases resulted in a standardized rate ratio of 4.87 (95\% confidence interval: 2.43-8.71). Impaired mismatch repair expression and microsatellite instability were seen in one out of two prostate cancer specimens available for testing.

Conclusion: Males with Lynch syndrome had a nearly fivefold increased risk of developing prostate cancer but did not appear to have earlier onset or a more aggressive phenotype.

Genet Med advance online publication 16 January 2014

Key Words: colorectal cancer; Lynch syndrome; microsatellite instability; mismatch repair deficiency; prostate cancer with LS. ${ }^{6,11}$ Other studies have focused on patients with hereditary prostate cancer and have looked for signs of MSI or lack of MMR proteins on IHC. One study found that 3 out of 77 prostate tumors from patients with hereditary prostate cancer harbor MSI. ${ }^{12}$ Another study found that 3 out of 31 patients with prostate cancer and a family history of CRC had MSI (two high and one low). IHC confirmed that the MSH2 and MSH6 proteins were missing in one prostate cancer case in which the patient was found to have a $\mathrm{MSH} 2$ mutation. ${ }^{13}$ A recent study found $65 \%$ of breast cancer tumors from LS patients to lack MMR, indicating that a double hit to MMR genes can potentially lead to breast cancer development. ${ }^{14}$

Prostate cancer is the most common cancer in males in the Western population. Although prostate cancer cells often contain somatic mutations, gene deletions, gene amplifications, chromosomal rearrangements (such as the fusion of the TMPRSS2 (transmembrane protease, serine 2) and the ETS (E26 transformation-specific) transcription factor genes), ${ }^{15}$ and hypermethylation of GSTP1 (glutathione S-transferase pi gene) at the time of diagnosis, no gatekeeper mutations that are consistently associated with prostate cancer have been found. ${ }^{16}$ Finding an increased incidence of prostate cancer in LS could potentially alter screening guidelines in these patients to allow for earlier detection. Currently, prostate cancer screening

${ }^{1}$ Division of Medical Oncology, Department of Internal Medicine, The Ohio State University Medical Center, Columbus, Ohio, USA; ${ }^{2}$ Division of Human Genetics, Department of Internal Medicine, The Ohio State University Medical Center, Columbus, Ohio, USA; ${ }^{3}$ Center for Biostatistics, The Ohio State University Medical Center, Columbus, Ohio, USA; ${ }^{4}$ Department of Pathology, The Ohio State University Medical Center, Columbus, Ohio, USA; ${ }^{5}$ Molecular Biology and Cancer Genetics Program, The Ohio State University Medical Center, Columbus, Ohio, USA. Correspondence: Richard M. Goldberg (richard.goldberg@osumc.edu) 
guidelines for LS patients are the same as those for the general population. The American Cancer Society recommends involving men in the decision-making process and if they decide to be screened, then to start prostate-specific antigen testing with or without digital rectal examination at 50 years of age. ${ }^{17}$

The objective of this cohort study was to assess whether the incidence of prostate cancer is increased above that of the general population in patients with LS by looking at all male patients diagnosed with LS at the Ohio State University (OSU).

\section{Study sample}

\section{MATERIALS AND METHODS}

All male patients diagnosed with a mutation in one of the MMR genes (MLH1, MSH2, MSH6, or PMS2) from June 1998 until June 2012 at the OSU were included in this study. This cohort consisted of patients previously included in the Ohio Lynch Syndrome study, ${ }^{18,19}$ patients included in the PMS2 study, ${ }^{20}$ and patients referred to the genetics clinic for counseling.

The Ohio Lynch Syndrome Project was a prospective cohort study that included 1,566 consecutive colon cancer patients diagnosed in any of the major metropolitan hospitals in Columbus, Ohio. Its purpose was to investigate the incidence of LS. ${ }^{18}$ The PMS2 study investigated 99 patients whose tumors lacked PMS2 protein expression on IHC (55 from the OSU, the remaining 44 through research collaborations in Newfoundland, Sweden, the National Cancer Institute-funded Colon Cancer Family Registry, and others), wherein the purpose was to investigate mutations in PMS2 with a new method using long-range polymerase chain reaction. ${ }^{20,21}$

\section{Data collection}

Baseline information on demographics and personal and family history of cancer, including histology reports, were obtained at enrollment in the study or at first visit to the genetics clinic. Patients who had not been seen at the genetics clinic in the 12 months before June 2012 were contacted, and changes to their cancer history were documented.

Follow-up time was defined as the time between detection of the first index cancer, entry into the LS studies, or the first visit to the genetics clinic (whichever came first) until the last date of follow-up or date of death.

\section{Mutation testing, immunohistochemistry, and microsatellite testing}

The methods used for germ-line mutation analysis have been previously described in detail. ${ }^{18}$ DNA was obtained from blood or normal colon tissue and was directly sequenced with the use of primers. The sequencing of the MLH1, MSH2, and MSH6 genes covered the promoter regions ( $\mathrm{MLH1}$ and $\mathrm{MSH} 2$ only), exons, and the intronic regions adjacent to all splice sites. For PMS2, conversion to haploidy (GMP Genetics, Waltham, MA) and western blot analysis were used in addition to sequencing. ${ }^{18}$ Multiplex ligation-dependent probe amplification assay of MLH1, MSH2, MSH6, or PMS2 was used if needed (if gene sequencing did not identify a mutation).
Where tumor tissue was available, we stained for MLH1 (Novacastra, Newcastle, UK), MSH2 (Calbiochem, Darmstadt, Germany), MSH6 (Epitomics, Burlingame, CA), and PMS2 (BD Pharmingen, San Jose, CA) proteins with IHC. Microsatellite testing was performed using five mononucleotide markers (BAT-25, BAT-26, NR-21, NR-24, and MONO-27) testing both tumor tissue and unaffected tissue with the Promega fluorescent multiplex polymerase chain reaction kit (Promega, Madison, WI). Results were considered positive when an allelic shift was present in the tumor compared with the unaffected tissue by two or more markers tested.

\section{Statistical analysis}

Descriptive statistics (mean, SD, median, range for continuous variables, and frequency for categorical variables) were provided to describe the patient population. SAS for Windows version 9.2 was used (SAS Institute, Cary, NC).

The incidence of prostate cancer was adjusted for age (in cohorts of 5 years) and race and compared with that of the Surveillance, Epidemiology, and End Results registry for 1999-2009. ${ }^{22}$ A standardized rate ratio was obtained by

\section{Table 1 Patient characteristics and mutation analysis}

\begin{tabular}{|c|c|c|}
\hline & $\begin{array}{l}\text { All patients, } \\
n=188(\%)\end{array}$ & $\begin{array}{c}\text { Prostate cancer Dx, } \\
\quad n=11(\%)\end{array}$ \\
\hline Median age (years) ${ }^{a}$ & $\begin{array}{c}46 \\
(\mathrm{Q} 1 ; \mathrm{Q} 4=33 ; 56)\end{array}$ & $\begin{array}{c}61 \\
(\mathrm{Q} 1 ; \mathrm{Q} 4=59 ; 71)\end{array}$ \\
\hline \multicolumn{3}{|l|}{ Race } \\
\hline Caucasian & $176(95.1 \%)$ & $11(100 \%)$ \\
\hline African American & $7(3.8 \%)$ & 0 \\
\hline Asian & $2(1.1 \%)$ & 0 \\
\hline Hispanic & 0 & 0 \\
\hline \multicolumn{3}{|l|}{ MMR gene mutation } \\
\hline MLH1 & $51(27.1 \%)$ & $1(9.1 \%)$ \\
\hline MSH2 & $87(46.3 \%)$ & $7(63.6 \%)$ \\
\hline MSH6 & $24(12.8 \%)$ & $2(18.2 \%)$ \\
\hline PMS2 & $26(13.8 \%)$ & $1(9.1 \%)$ \\
\hline \multicolumn{3}{|l|}{ MMR mutation type } \\
\hline Del/ins & $47(25 \%)$ & $2(18.1 \%)$ \\
\hline Large genomic del/dup & $40(21.3 \%)$ & $4(36.5 \%)$ \\
\hline Missense & $25(13.3 \%)$ & 0 \\
\hline Nonsense & $25(13.3 \%)$ & $2(18.1 \%)$ \\
\hline At splice site & $51(27.1 \%)$ & $3(27.3 \%)$ \\
\hline \multicolumn{3}{|l|}{ Location of index cancer } \\
\hline Colonic & $77(41 \%)$ & $5(45.5 \%)$ \\
\hline Extracolonic & $26(13.8 \%)$ & $6(54.5 \%)$ \\
\hline No cancer diagnosed & $85(45.2 \%)$ & N/A \\
\hline $\begin{array}{l}\text { Mean follow-up time } \pm \\
\text { SD (years) }\end{array}$ & $6.2 \pm 7.2$ & $6.4 \pm 4.8$ \\
\hline
\end{tabular}

Dx, diagnosed; MMR, mismatch repair; $n$, number; N/A, not applicable; Q, quartile; $\mathrm{SD}$, standard deviation.

${ }^{a}$ At MMR mutation diagnosis. 
comparing the observed rate with the expected rate by the direct method of standardization. Fisher's exact test was used to compare the incidence of MMR gene mutations in patients with prostate cancer versus that in subjects without prostate cancer. Pathology reports for the prostate cancer diagnosis were obtained when possible.

\section{RESULTS}

\section{Patient and mutation characteristics}

One hundred and eighty eight males (median age: 46 years, Q1;Q4: 33;56, range: 11-83) were identified as MMR mutation carriers either by testing (95\%) or as obligate carriers (5\%) in 90 families. Their mean follow-up time was 6.2 years (SD: \pm 7.2 years), with a total of 1,156 person-years. Table 1 includes further details on patient characteristics, gene mutation, and index cancers.

Table 2 Characteristics of patients with prostate cancer

\begin{tabular}{lclll}
$\begin{array}{l}\text { Patient } \\
\text { no. }\end{array}$ & $\begin{array}{c}\text { Age at } \\
\text { diagnosis } \\
\text { (years) }\end{array}$ & $\begin{array}{c}\text { Stage at } \\
\text { diagnosis }\end{array}$ & Gene & \multicolumn{1}{c}{ Mutation } \\
\hline 1 & 64 & Localized & MLH1 & Del exon 19 \\
\hline 2 & 68 & Not available & MSH2 & C.942+3A>T \\
\hline 3 & 56 & Metastatic & MSH2 & C.942+3A>T \\
\hline $4^{\text {a }}$ & 65 & $\begin{array}{l}\text { Locally } \\
\text { advanced }\end{array}$ & MSH2 & Del exons 1-6 \\
\hline 5 & 55 & Localized & MSH2 & C.942+3A>T \\
\hline $6^{a}$ & 57 & Localized & MSH2 & Del exon 8 \\
\hline 7 & 55 & Not available & MSH2 & C.2038C>T \\
\hline 8 & 71 & Localized & MSH2 & C.1906G>C \\
\hline 9 & 82 & Localized & MSH6 & C.3155_3156delAG \\
\hline 10 & 74 & Not available & MSH6 & c.3261dupC \\
\hline 11 & 63 & Not available & PMS2 & Del exons 11-12 \\
\hline
\end{tabular}

aHad available prostate cancer tissue for immunohistochemistry and microsatellite instability testing.

\section{Prostate cancer incidence}

Eleven patients (5.9\%) were diagnosed with prostate cancer during the study period (Tables 1 and 2). In two cases, prostate cancer was the index cancer; in two other cases, it was found at the same time as another cancer; and in seven cases, the diagnosis of prostate cancer was preceded by that of other cancers. One patient was identified as an MLH1 carrier, seven as MSH2 carriers, two as MSH6 carriers, and one as a PMS2 carrier. There was no significant difference between the incidence of gene mutations in patients with prostate cancer versus that in subjects without prostate cancer (MLH1: $9.1 \%$ in patients with prostate cancer versus $28.3 \%$ in subjects without prostate cancer, $P=0.29$; $M S H 2: 63.6$ vs. $45.2 \%, P=0.35$; MSH6: 18.2 vs. $12.4 \%, P=0.64$; $P M S 2: 9.1$ vs. $14.1 \%, P=1.0$ ). The observed rate of 11 cases was compared with that of the expected 2.26 cases, and the standardized rate ratio was calculated at 4.87 (95\% confidence interval (CI): 2.43-8.71).

Five patients $(72 \%)$ were diagnosed with localized disease, one (14\%) with locally advanced disease, and one (14\%) with metastatic disease (Table 2). In four cases, the stage at diagnosis was unknown. The median age at diagnosis was 64 years. All of the prostate cancer patients had known pathogenic MMR mutations, with no cases of missense mutations. Seven out of 11 patients had not received radiation therapy to the pelvis before their prostate cancer diagnosis; in the other 4 cases, we did not have information on previous treatment.

\section{Immunohistochemistry and microsatellite testing}

Two tumors were available for IHC and MSI testing. Patient 4 (Table 2) was found to have MSH2/MSH6 missing on IHC, in addition to MSI in the prostate tumor. Patient 6 was found to have all MMR proteins intact on IHC, and the tumor was microsatellite stable.

\section{Other cancers}

One hundred and three patients were diagnosed with other cancers: 73 (38.9\%) had 1 primary tumor during the study

Table 3 Previous studies looking at prostate cancer incidence in Lynch syndrome

\begin{tabular}{|c|c|c|c|c|c|c|}
\hline Reference & Place & $N(\mathrm{PrCa})$ & SIR/SRR & $95 \% \mathrm{Cl}$ & Median age at Dx & Gene involved \\
\hline Aarnio et al. ${ }^{2 a}$ & Finland & $360(4)$ & SIR 2.9 & $0.8-7.4$ & NR & NR \\
\hline Scott et al. ${ }^{10}$ & Wales & 95 families (1) & SIR 1.02 & $0.1-13.6$ & NR & MLH1 (0), MSH2 (1) \\
\hline Grindedal et al. ${ }^{6}$ & Norway & $106(9)$ & SIR 5.9 & $4.1-17.1$ & 60.4 & $\begin{array}{l}\text { MLH1 (0), MSH2 (6), } \\
\text { MSH6 (2), PMS2 (1) }\end{array}$ \\
\hline Win et al. ${ }^{5 b}$ & $\begin{array}{l}\text { Australia, Canada, } \\
\text { United States }\end{array}$ & $382(19)$ & SIR 2.05 & $1.23-3.01$ & 64 & $\begin{array}{l}\text { MLH1 (3), MSH2 (15), } \\
\text { MSH6 (1), PMS2 (0) }\end{array}$ \\
\hline Pande et al. ${ }^{9}$ & MD Anderson & $151(3)$ & SIR 0.93 & $0.19-2.7$ & 65 & NR \\
\hline Engel et al. ${ }^{8}$ & Germany, The Netherlands & $1,107(17)$ & SIR 2.5 & $1.4-4.0$ & 59 & NR \\
\hline Raymond et al. ${ }^{7}$ & University of Michigan & $412(15)$ & HR 1.99 & $1.31-3.03$ & 65 & NR \\
\hline This study & The Ohio State University & $188(11)$ & SRR 4.87 & $2.43-8.71$ & 64 & $\begin{array}{l}\text { MLH1 (1), MSH2 (7), } \\
\text { MSH6 (2), PMS2 (1) }\end{array}$ \\
\hline
\end{tabular}

$\mathrm{Cl}$, confidence interval; Dx, diagnosis; HR, hazard ratio; N, number; NR, not reported; PrCa, prostate cancer; SIR, standardized incidence ratio; SRR, standardized rate ratio. astudied MLH1 (47 families) and MSH2 (3 families). bStudied patients who had a previous history of colorectal cancer. 
period, 22 (11.7\%) had 2 primary tumors, 7 (3.7\%) had 3 primary tumors, and $1(0.5 \%)$ had 4 primary tumors. The origin of the index cancer was colorectal in 77 (41.0\%), dermal in 8 (4.3\%), ureteral in $5(2.7 \%)$, upper gastrointestinal in $3(1.6 \%)$, prostatic in $2(1.1 \%)$, and of the brain and other cancers in, respectively, $2(1.1 \%)$ and $6(3.2 \%)$ patients.

\section{DISCUSSION}

Prostate cancer incidence was significantly increased-almost fivefold-in this relatively large cohort of LS patients. This is in concordance with the results of most other studies (Table 3) that have demonstrated an increased risk, with standardized incidence ratios (SIRs) ranging from 2.0 to 5.9.

The median age of diagnosis for prostate cancer in the Surveillance, Epidemiology, and End Results registry for the period 2005-2009 was 67 years, with $80 \%$ having localized disease at diagnosis. ${ }^{23}$ Our cohort shows consistent results, with a median age of 64 years and $71 \%$ with localized disease at diagnosis, and therefore it does not appear that prostate cancer is being diagnosed at a younger age or at a more advanced stage in LS patients compared with the general population. The incidence was adjusted for both age and race to eliminate the confounding effect of the young age of our cohort on prostate cancer incidence.

Mutations in $\mathrm{MSH} 2$ appear to be more common in patients who are diagnosed with prostate cancer, and although not statistically significant in our study, most mutations in patients with prostate cancer occurred in MSH2 (63.6\%). Win et al. ${ }^{5}$ showed an increased incidence of prostate cancer in patients with previous colon cancer diagnosis (SIR: 2.11 ; 95\% CI: $1.22-$ 3.14) but not in those with previous rectal cancer (SIR: 0.88; 95\% CI: 0-3.36). When grouped by gene mutation, only $\mathrm{MSH} 2$ mutations were found to increase the risk of prostate cancer (SIR: 3.62, 95\% CI: 2.07-5.36). ${ }^{5}$ Aarnio et al. ${ }^{2}$ looked at Finnish families, in which the most common mutation occurs in the MLH1 gene. In fact, 47 of the 50 families included in this study had a mutation in the MLH1 gene, which could potentially explain why prostate cancer was not increased in this cohort. Goecke et al. ${ }^{24}$ found 8 cases of prostate cancer in 333 males carrying the $\mathrm{MSH} 2$ mutation in a population-based study performed in Germany. SIR was not reported, but they noted that there was a marginal association between $\mathrm{MSH} 2$ mutations and prostate cancer and determined that the median age of diagnosis in their cohort was 59 years. Similarly, an increased risk in only $\mathrm{MSH} 2$ mutation carriers was seen in a cohort study conducted in the United Kingdom. ${ }^{25}$ It is therefore important to be mindful of the ratio of mutations in the four MMR genes when comparing incidence of cancers across LS studies because they may vary in different populations.

Urothelial carcinoma was found in three patients who were also diagnosed with prostate cancer in our study; this could potentially be due to the association with $\mathrm{MSH} 2$ mutations, which are also known to increase the incidence of urothelial carcinoma, or due to heightened awareness of genitourinary malignancies in patients with a previous diagnosis of a genitourinary cancer.
A study on pooled cohorts from the national LS registries in Germany and the Netherlands revealed an increased incidence, with SIR $=2.5$ (95\% CI: 1.4-4.0), wherein patients with MSH2 mutations had an increased risk of developing urothelial and prostate cancer. Median age at diagnosis was 59 years (range: $50-74)$. The cumulative risk at age 70 was $9.1 \%(95 \%$ CI: $4.4-13.8){ }^{8}$

The two patients who provided prostate tumor tissue for testing had mixed results, with the prostate tumor from patient 4 (deletion of exons 1-6 in MSH2) exhibiting both loss of protein expression and MSI, whereas the prostate tumor from patient 6 (deletion of exon 8 in MSH2) had intact protein expression and microsatellite stability. We conclude that although impaired MMR function may contribute to prostate cancer development in some cases, it is unlikely to do so in all cases.

Although several studies have looked at MMR protein expression by IHC in prostate cancer, no study has looked at the number of accumulated mutations in combination with MMR system inactivation. If MMR protein inactivation (by germ-line mutations) is responsible for prostate cancer development, one might hypothesize that this would happen by the same mechanism, by accumulation of multiple mutations from uncorrected DNA repair errors, leading to a hypermutated phenotype such as the one seen in CRCs related to LS.

The strengths of our study include the fact that all patients had confirmed mutational diagnosis, except for $5 \%$ who were diagnosed as obligate carriers with subsequent-generation family members being diagnosed with LS by mutation analysis. Moreover, the study includes consecutive CRC patients enrolled in the Ohio Lynch Syndrome Project; therefore, the cohort has a representation of unselected patients who did not seek care at a genetics clinic, somewhat limiting selection bias. Limitations in our study include patients lost to follow-up (who had to be censored prematurely) and the relatively young age of the cohort.

Our study strengthens current growing evidence that the incidence of prostate cancer is increased in males with LS. We believe that heightened surveillance should be considered in these cases, especially in patients carrying a mutation in MSH2. The American Cancer Society currently recommends beginning discussions of screening at age $40-45$ in patients at high risk (e.g., African-American men and men with a firstdegree relative with prostate cancer $<65$ years of age) ${ }^{17}$ but because prostate cancer does not seem to develop earlier in patients with LS and it is not more aggressive, it is unclear whether these high-risk guidelines are applicable to this group. The IMPACT study, a multicenter observational study initiated in the United Kingdom, is currently investigating the impact of prostate cancer screening on 40 - to 69 -year-old men with mutations in the breast cancer genes BRCA1/2 and LS mutations. Patients are offered annual prostate-specific antigen checks, and the threshold for prostatic biopsy is a prostate-specific antigen level $>3.0 \mathrm{ng} / \mathrm{ml}$. $^{26}$ This study may answer current questions about the benefit of surveillance in these patients. 


\section{DISCLOSURE}

R.M.G. holds a consultant role with Bayer (uncompensated), Jennerex (uncompensated), Lilly (compensated), Pfizer (compensated), and Sanofi (uncompensated) and has received honoraria from Sanofi and research funding from Bayer and Sanofi. H.H. has received honoraria and research funding from Myriad Genetic Laboratories. The other authors declare no conflict of interest.

\section{REFERENCES}

1. Comprehensive molecular characterization of human colon and rectal cancer. Nature 2012;487:330-337.

2. Aarnio M, Sankila R, Pukkala E, et al. Cancer risk in mutation carriers of DNAmismatch-repair genes. Int J Cancer 1999;81:214-218.

3. Järvinen HJ, Aarnio M, Mustonen $\mathrm{H}$, et al. Controlled 15-year trial on screening for colorectal cancer in families with hereditary nonpolyposis colorectal cancer. Gastroenterology 2000;118:829-834.

4. Schmeler KM, Lynch HT, Chen LM, et al. Prophylactic surgery to reduce the risk of gynecologic cancers in the Lynch syndrome. N Engl J Med 2006;354: 261-269.

5. Win AK, Lindor NM, Young JP, et al. Risks of primary extracolonic cancers following colorectal cancer in lynch syndrome. J Nat/ Cancer Inst 2012;104:1363-1372.

6. Grindedal EM, Møller P, Eeles R, et al. Germ-line mutations in mismatch repair genes associated with prostate cancer. Cancer Epidemiol Biomarkers Prev 2009;18:2460-2467.

7. Raymond VM, Mukherjee $B$, Wang $F$, et al. Elevated risk of prostate cancer among men with Lynch syndrome. J Clin Oncol 2013;31:1713-1718.

8. Engel $C$, Loeffler $M$, Steinke $V$, et al. Risks of less common cancers in proven mutation carriers with lynch syndrome. J Clin Oncol 2012;30:4409-4415.

9. Pande $\mathrm{M}$, Wei $\mathrm{C}$, Chen J, et al. Cancer spectrum in DNA mismatch repair gene mutation carriers: results from a hospital based Lynch syndrome registry. Fam Cancer 2012:11:441-447.

10. Scott RJ, McPhillips M, Meldrum CJ, et al. Hereditary nonpolyposis colorectal cancer in 95 families: differences and similarities between mutation-positive and mutation-negative kindreds. Am J Hum Genet 2001;68:118-127.

11. Soravia $\mathrm{C}$, van der Klift $\mathrm{H}$, Bründler MA, et al. Prostate cancer is part of the hereditary non-polyposis colorectal cancer (HNPCC) tumor spectrum. Am J Med Genet A 2003;121A:159-162.
12. Ahman AK, Jonsson BA, Damber JE, Bergh A, Grönberg H. Low frequency of microsatellite instability in hereditary prostate cancer. BJU Int 2001;87:334-338.

13. Bauer CM, Ray AM, Halstead-Nussloch BA, et al. Hereditary prostate cancer as a feature of Lynch syndrome. Fam Cancer 2011;10:37-42.

14. Lotsari JE, Gylling A, Abdel-Rahman WM, et al. Breast carcinoma and Lynch syndrome: molecular analysis of tumors arising in mutation carriers, noncarriers, and sporadic cases. Breast Cancer Res 2012;14:R90.

15. Tomlins SA, Rhodes DR, Perner $S$, et al. Recurrent fusion of TMPRSS2 and ETS transcription factor genes in prostate cancer. Science 2005;310:644-648.

16. Nelson WG, De Marzo AM, Isaacs WB. Prostate cancer. N Engl J Med 2003;349:366-381

17. Wolf AM, Wender RC, Etzioni RB, et al.; American Cancer Society Prostate Cancer Advisory Committee. American Cancer Society guideline for the early detection of prostate cancer: update 2010. CA Cancer J Clin 2010;60:70-98.

18. Hampel H, Frankel WL, Martin E, et al. Screening for the Lynch syndrome (hereditary nonpolyposis colorectal cancer). N Engl J Med 2005;352:18511860

19. Hampel H, Frankel WL, Martin E, et al. Feasibility of screening for Lynch syndrome among patients with colorectal cancer. J Clin Oncol 2008;26:57835788

20. Clendenning M, Senter L, Hampel $H$, et al. A frame-shift mutation of PMS2 is a widespread cause of Lynch syndrome. J Med Genet 2008;45:340-345.

21. Senter L, Clendenning M, Sotamaa K, et al. The clinical phenotype of lynch syndrome due to germ-line PMS2 mutations. Gastroenterology 2008;135:419428.e411.

22. United States Department of Health and Human Services CfDCaPaNCI. United States Cancer Statistics: 1999 - 2009 Incidence, WONDER Online Database. http://wonder.cdc.gov/cancer-v2009.html Accessed 25 May 2013

23. Howlader N, Noone A, Krapcho M, et al. SEER Cancer Statistics Review, 19752009 (Vintage 2009 Populations), National Cancer Institute. Bethesda, MD. http://seer.cancer.gov/csr/1975_2009_pops09/. 2012; based on November 2011 SEER data submission, posted to the SEER web site, April 2012.

24. Goecke T, Schulmann K, Engel C, et al.; German HNPCC Consortium. Genotype-phenotype comparison of German MLH1 and MSH2 mutation carriers clinically affected with Lynch syndrome: a report by the German HNPCC Consortium. J Clin Oncol 2006;24:4285-4292.

25. Barrow PJ, Ingham S, O'Hara C, et al. The spectrum of urological malignancy in Lynch syndrome. Fam Cancer 2013;12:57-63.

26. IMPACT. Targeted Prostate Cancer Screening Study. http://www.impact-study. co.uk/. Accessed 8 June 2013. 